\title{
Increased expression of insulin-like growth factor-binding protein-3 is implicated in erectile dysfunction in two-kidney one-clip hypertensive rats after propranolol treatment
}

\author{
Zhang-Yan Zhou*, Zhong-Hua Yang, Xing-Huan Wang, Hong Cao*, Dong Chen, Yong-Zhi Wang, \\ Hai-Hong Zhou, Mou Peng, Quan-Liang Liu and Shao-Ping Wan
}

This study aimed to investigate the role of insulin-like growth factor-binding protein-3 (IGFBP-3) in erectile dysfunction (ED) in two-kidney one-clip $(2 \mathrm{~K}-1 \mathrm{C})$ hypertensive rats treated with the $\beta$-blocking agent propranolol. Adult male Wistar rats were randomly divided into three groups: a normal control group, a hypertensive control group and a propranolol treatment group ( $n=9$ ). After 4 weeks of propranolol treatment, intracavernous pressure (ICP) responses to electrical stimulation of the cavernous nerves were evaluated. The expression of IGFBP-3 and insulin-like growth factor-1 (IGF-1) mRNA and protein in the rat cavernous tissue were detected by quantitative real-time PCR and Western blot, respectively. The concentration of cyclic guanosine monophosphate (cGMP) in the cavernous tissue was determined by enzyme-linked immunosorbent assay (ELISA). Cavernosal pressure in response to cavernous nerve stimulation was decreased 4 weeks after propranolol treatment $(\boldsymbol{P}<0.01$, compared to the hypertensive control group). IGFBP-3 mRNA and protein expression was increased in the propranolol treatment group compared to the hypertensive control group $(P<0.01)$, whereas IGF-1 expression was decreased in the propranolol treatment group compared to the hypertensive control group $(\boldsymbol{P}<0.01)$. In addition, cavernous cGMP concentration was decreased in the propranolol treatment group compared to the hypertensive control group $(\boldsymbol{P}<0.01)$. Taken together, these results suggest that the upregulation of IGFBP-3 may play a role in the development of ED in hypertensive rats. Asian Journal of Andrology (2011) 13, 851-855; doi:10.1038/aja.2011.99; published online 29 August 2011

Keywords: erectile dysfunction; insulin-like growth factor-binding protein-3; propranolol; two-kidney one-clip hypertension

\section{INTRODUCTION}

Erectile dysfunction (ED) is a multifactorial disease that is highly prevalent in hypertensive patients treated with $\beta$-blocking agents. ${ }^{1,2}$ $\beta$-blockers have been associated with ED in up to $20 \%$ of men who use these agents for the treatment of hypertension. ${ }^{3}$ A large body of evidence has shown that nitric oxide (NO) production is increased as a buffer against hypertension in two-kidney one-clip (2K-1C) hypertensive animal models. ${ }^{4-6}$ The increase in NO production that is observed in several vascular beds from $2 \mathrm{~K}-1 \mathrm{C}$ hypertensive animals may not completely prevent high blood pressure but could serve as a protective vascular mechanism that contributes to the preservation of the erectile function in this model. ${ }^{7}$

Insulin-like growth factor-binding protein-3 (IGFBP-3) is a member of the insulin-like growth factor (IGF) family. A previous study has shown that ED was correlated with the increased expression of IGFBP3 in patients with diabetes mellitus, ${ }^{8}$ suggesting a potential role for IGFBP-3 in ED. In this study, we employed $2 \mathrm{~K}-1 \mathrm{C}$ hypertensive rats as a model to investigate the expression of IGFBP-3 at both the mRNA and protein levels in this system.

\section{MATERIALS AND METHODS}

Experimental animals

Twenty-seven adult male Wistar rats (grade SPF, 3-month-old, 310-330 g) were obtained from the Hubei Laboratory Animal Research Center (Wuhan, China). The rats were randomly divided into three groups: a normal control group, a hypertensive control group and a propranolol treatment group $(n=9$, in each group). Eighteen $2 \mathrm{~K}-1 \mathrm{C}$ hypertensive rats were prepared as previously described. $^{7}$ Briefly, rats were anaesthetised by ketamine/xylazine (87 and $13 \mathrm{mg} \mathrm{kg}^{-1}$, respectively), and a silver clip $(0.2 \mathrm{~mm}$ internal diameter) was placed on the left renal artery. Nine normal control rats were subjected to a similar procedure, but no silver clip was placed. Four weeks after the surgery, DL-propranolol was dissolved in the drinking water at a concentration of $600 \mathrm{mg} \mathrm{l}^{-1}$ and administered to the rats in the propranolol treatment group by oral gavage (approximately $30 \mathrm{mg}$ per rat per day ), as recommended in a previous study. ${ }^{9}$ Chronic treatment lasted for 4 weeks. The rats in the two control groups were given citrate buffer $\left(100 \mathrm{mmol}^{-1}\right.$ citric acid and $200 \mathrm{mmol}^{-1}$ disodium phosphate, $\mathrm{pH} 7.0$ ) by oral

Department of Urology, Zhongnan Hospital of Wuhan University, Wuhan 430071, China

* These authors contributed equally to this work.

Correspondence: Dr XH Wang (urologistwxh@gmail.com)

Received: 2 February 2011; Revised: 11 May 2011; Accepted: 29 June 2011; Published online: 29 August 2011 
gavage (50 ml per rat). Systolic blood pressure (SBP) was measured weekly with a tail-cuff, and animals were considered hypertensive if SBP was higher than $160 \mathrm{mmHg}$ at the end of the eighth week after silver clip implantation or sham surgery. The animal experiments were approved by the Wuhan University Animal Care and Use Committee (Wuhan, China).

\section{Measurement of erectile response}

Erectile function was assessed by measuring intracavernous pressure (ICP) following electrostimulation of cavernous nerves after 4 weeks of propranolol treatment, as previously described. ${ }^{10}$ Mean arterial pressure (MAP) and ICP lines were connected to a pressure transducer, which was then connected via a transducer amplifier to a data acquisition board (RM6240; Chengdu Instrument, Chengdu, China), and electrical stimulation of the cavernous nerves ( $1 \mathrm{~ms}$ pulse, $60 \mathrm{~s}$, $15 \mathrm{~Hz}, 2.5 \mathrm{~V}$ ) was performed. The ratio of maximal ICP-to-MAP (ICP/ $\mathrm{MAP}$ ) and total ICP (the area under curve) were recorded for each rat. After measurement of the erectile responses, all rats were killed with an intraperitoneal overdose of pentobarbital $\left(80 \mathrm{mg} \mathrm{kg}^{-1}\right)$, and the penile shaft was removed for further analyses.

\section{Quantitative real-time PCR analysis}

Total RNA was extracted from rat penile samples using TRIzol (Invitrogen, Merelbeke, Belgium) according to the manufacturer's protocol, resuspended in RNase-free water and stored at $-80{ }^{\circ} \mathrm{C}$. IGFBP-3 and IGF-1 mRNA levels were analysed by MyiQ single colour real-time PCR detection system (Bio-Rad Laboratories, Hercules, CA, USA) with the following primers: IGFBP-3, forward $5^{\prime}$-AGCCGTCTCCTGGAAACACC-3' and reverse 5'-CCCGCTTTCTGCCTTTGG-3'; IGF-1, forward 5'-GACATGCCCAAGACCCAGAAGGA-3' and reverse 5' -CGGTGGCATGTCACTCTTCACTC-3'. Quantitative mRNA measurements were performed in triplicate and normalised to an internal control (glyceraldehyde 3-phosphate dehydrogenase). Data were analysed with ABI 7300 SDS software (Applied Biosystems, Foster City, CA, USA).

\section{Western blot analysis}

Rat penile samples were lysed in radioimmunoprecipitation assay buffer $(1 \times$ Tris-buffered saline, $1 \%$ Nonidet P-40, 0.5\% sodium deoxycholate, $0.1 \%$ SDS, $0.004 \%$ sodium azide, $10 \mu \mathrm{ml}^{-1}$ phenylmethylsulfonyl fluoride, $10 \mu \mathrm{ml}^{-1}$ protease inhibitor cocktail, $10 \mu \mathrm{ml}^{-1}$ sodium orthovanadate). Protein concentration was measured using Coomassie Plus Protein Assay Reagent (Pierce Biotechnology, Rockford, IL, USA). Equal quantities $(30 \mu \mathrm{g})$ of lysates were separated on $10 \%$ sodium dodecyl sulphate polyacrylamide gels and electroblotted onto polyvinylidene fluoride membranes (Bio-Rad, Hercules, CA, USA). The membranes were blocked and then incubated with rabbit anti-IGFBP-3 antibody (Sc-9028) and rabbit antiIGF-I antibody (Sc-9013) (Santa Cruz Biotechnology, Santa Cruz, CA, USA), both at $1: 1000$ dilutions. Chemiluminescence was detected using enhanced chemiluminescence reagent (Amersham, Buckinghamshire, UK) and bands were visualised and quantitated by densitometry using the Mint Image analysis system (Shanghai Institute of Optical Instruments, Shanghai, China).

\section{Enzyme-linked immunosorbent assay (ELISA)}

The cyclic guanosine monophosphate (cGMP) concentration in rat penile tissue lysate was determined using an ELISA cGMP detection kit (R\&D Systems, Minneapolis, MN, USA) according to the manufacturer's instructions.

\section{Statistical analysis}

Data were expressed as mean \pm s.d. and analysed using the SPSS 12 package (SPSS Inc., Chicago, IL, USA). Multiple comparisons among groups were analysed by one-way ANOVA followed by a post hoc StudentNewman-Keuls tests. Differences were considered significant at $P<0.05$.

\section{RESULTS}

Propranolol attenuates erectile function in hypertensive rats We measured the ICP/MAP ratio and total ICP (the area under the curve) during electrostimulation of the cavernous nerve in three groups 4 weeks after propranolol treatment. Representative ICP traces are shown in Figure 1a-c. Electrostimulation elicited a lower ICP/ MAP ratio $(P<0.05$, Figure 1d) and total ICP $(P<0.01$, Figure 1e) in the hypertensive control group than in the normal control group. The propranolol treatment group also showed a lower ICP/MAP ratio (Figure 1d) and total ICP (Figure 1e) than the normal control group $(P<0.01$, respectively). In addition, the propranolol treatment group showed lower total ICP $(P<0.05$, Figure 1e) than the hypertensive control group. Taken together, these data suggest that propranolol treatment attenuated erectile function in hypertensive rats.

\section{Propranolol treatment upregulates IGFBP-3 expression in rat cavernous tissue}

Quantitative real-time PCR (real-time qPCR) analysis showed that the cavernous IGFBP-3 mRNA level in the propranolol treatment group was higher than that in the hypertensive control group $(P<0.01$, Figure 2). The cavernous IGFBP-3 mRNA level was lower in the hypertensive control group than in the normal control group $(P<0.01$, Figure 2).

Similarly, Western blot analysis showed that the cavernous IGFBP-3 protein level in the propranolol treatment group was higher than that in the hypertensive control group $(P<0.01$, Figure 3, lower panel), and the cavernous IGFBP-3 protein level was lower in the hypertensive control group than in the normal control group $(P<0.01$, Figure 3, lower panel). These results are consistent with the results of real-time qPCR analysis and demonstrate that propranolol treatment upregulates IGFBP-3 expression in rat cavernous tissue at both the mRNA and protein levels.

\section{Propranolol treatment downregulates IGF-1 expression in rat cavernous tissue}

Real-time qPCR analysis showed that the cavernous IGF-1 mRNA level in the propranolol treatment group was lower than that in the hypertensive control group ( $P<0.01$, Figure 4$)$. The cavernous IGF-1 mRNA level was higher in the hypertensive control group than in the normal control group $(P<0.01$, Figure 4).

Western blot analysis also showed that the cavernous IGF-1 protein level in the propranolol treatment group was lower than that in the hypertensive control group ( $P<0.01$, Figure 5, lower panel). The cavernous IGF-1 protein level was higher in the hypertensive control group than in the normal control group $(P<0.01$, Figure 5, lower panel). These results are consistent with the results of real-time qPCR analysis. Together, these results demonstrate that propranolol treatment downregulates IGF-1 expression in rat cavernous tissue at both the mRNA and protein levels.

Propranolol treatment leads to a decreased cGMP concentration in rat cavernous tissue

Because nitric oxide-cyclic guanosine monophosphate (NO-cGMP) signalling is crucial for penile erection, we measured the cGMP 

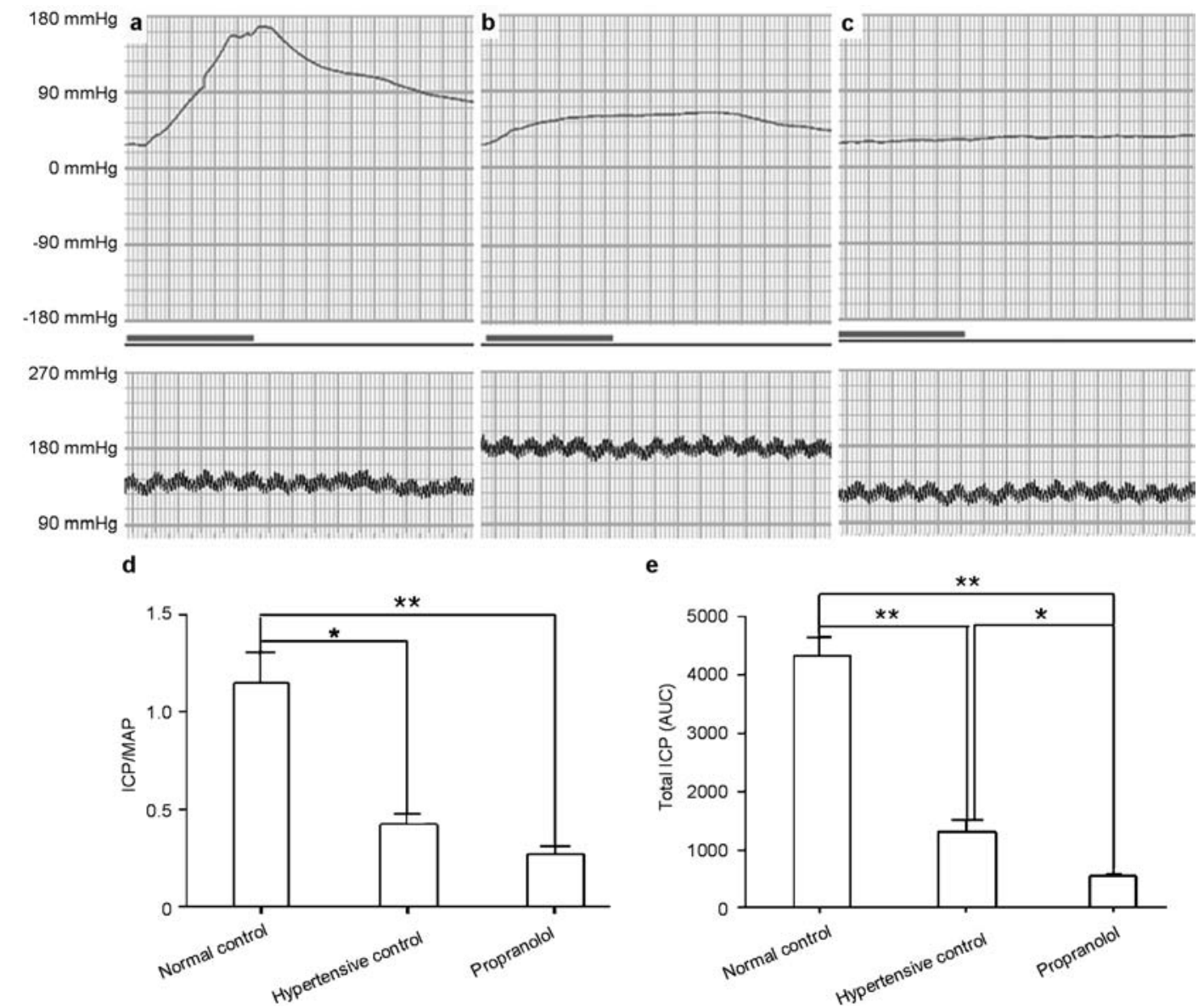

Figure 1 Propranolol attenuates erectile function in hypertensive rats. Representative ICP responses to the electrical stimulation of cavernous nerves 4 weeks after propranolol treatment in normal control (a), hypertensive control (b) and propranolol-treated rats (c) ( $n=9$, respectively). The bold line indicates 1 min of electrical stimulation to the cavernous nerves. ICP/MAP (d) and total ICP (the area under the curve, AUC) data (e) were collected from nine rats in each group. ${ }^{* *} P<0.01$, $* P<0.05$. ICP, intracavernous pressure; MAP, mean arterial pressure.

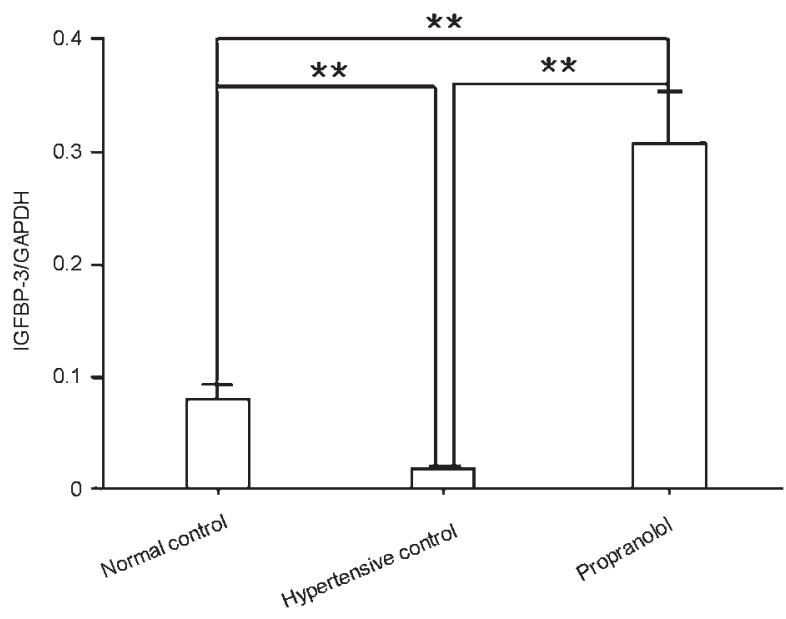

Figure 2 Propranolol increases IGFBP-3 mRNA expression in rat cavernous tissue. Quantitative real time-PCR analysis of IGFBP-3 mRNA levels in rat cavernous tissue was conducted 4 weeks after propranolol treatment. The data were collected from nine rats in each group. ${ }^{*} P<0.01$. GAPDH, glyceraldehyde 3 -phosphate dehydrogenase; IGFBP-3, insulin-like growth factor-binding protein-3. concentration in rat penile tissue by ELISA. Cavernous cGMP concentration was similar in the hypertensive control group and the normal control group $(P>0.05$, Figure 6). However, the cGMP concentration in penile tissue from the propranolol treatment group was decreased compared to the hypertensive control group 4 weeks after propranolol treatment $(P<0.01$, Figure 6), indicating that the disruption of NO-cGMP signalling may contribute to ED in hypertensive rats after propranolol treatment.

\section{DISCUSSION}

In the current study, we demonstrated an obvious increase in IGFBP- 3 expression in the penile tissue of $2 \mathrm{~K}-1 \mathrm{C}$ hypertensive rats 4 weeks after propranolol treatment, and that this increase is accompanied by a decrease in ICP. These data suggest that increased expression of IGFBP-3 could play a role in the development of ED in $2 \mathrm{~K}-1 \mathrm{C}$ hypertensive rats after propranolol treatment and that attenuated erectile responses may be associated with decreased IGF-1 and cGMP availability.

IGFBP-3 is the major IGFBP in the serum and transports 70\%-90\% of circulating IGFs. ${ }^{11}$ When binding to IGF-1, IGFBP-3 may modulate cell growth by limiting the bioavailability of IGF- $1 .{ }^{12}$ It has been confirmed that increased IGFBP-3 expression in diabetes attenuates the cellular response to IGF-1 through the high affinity binding of IGF-1 

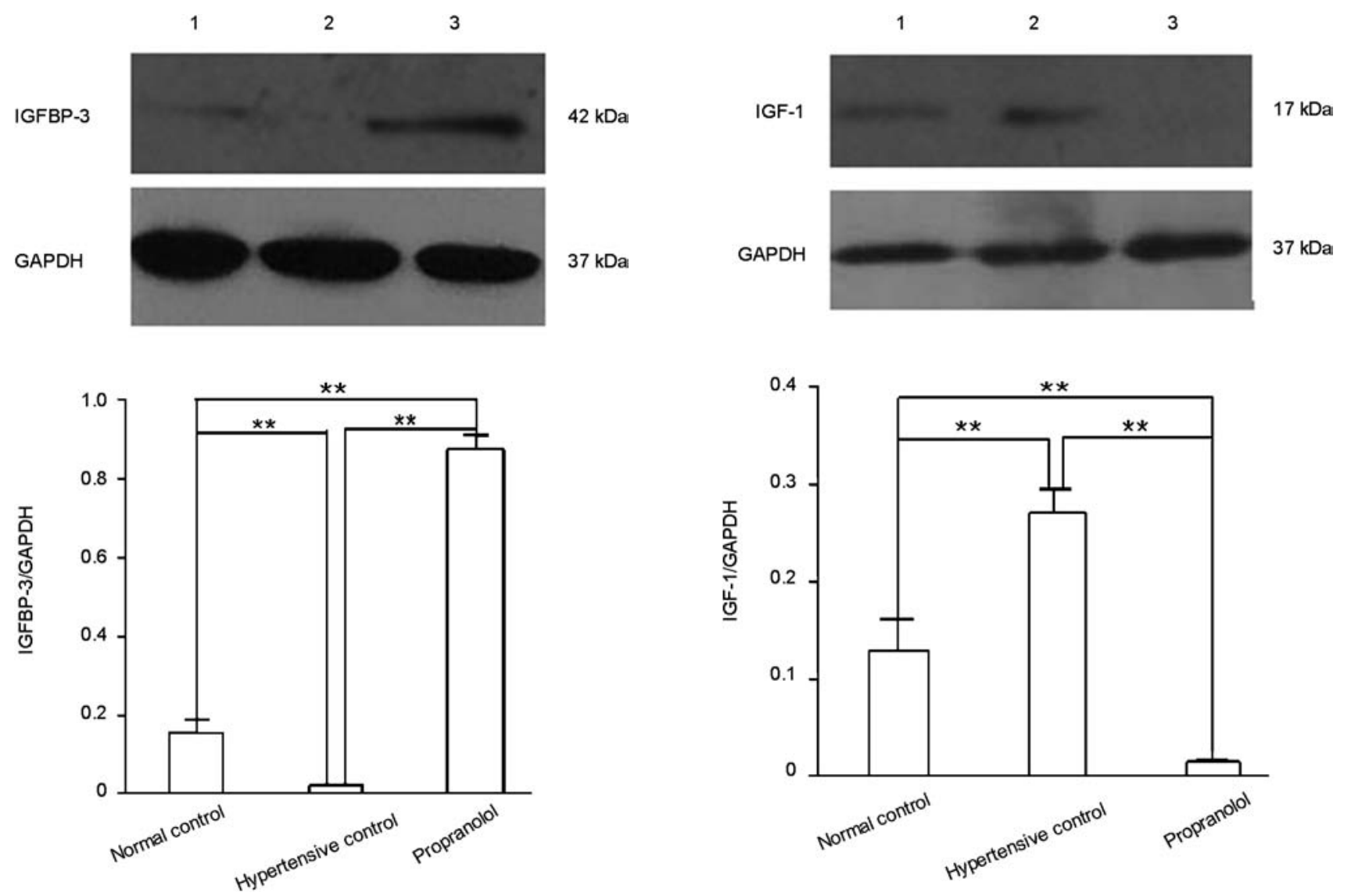

Figure 3 Propranolol increases IGFBP-3 protein expression in rat cavernous tissue. Western blot analysis of IGFBP-3 protein expression in cavernous tissue from normal control (lane 1), hypertensive control (lane 2) and propranololtreated rats (lane 3 ) was done 4 weeks after propranolol treatment. The data were collected from nine rats in each group. $* * P<0.01$. IGFBP-3, insulin-like growth factor-binding protein-3; GAPDH, glyceraldehyde 3-phosphate dehydrogenase.

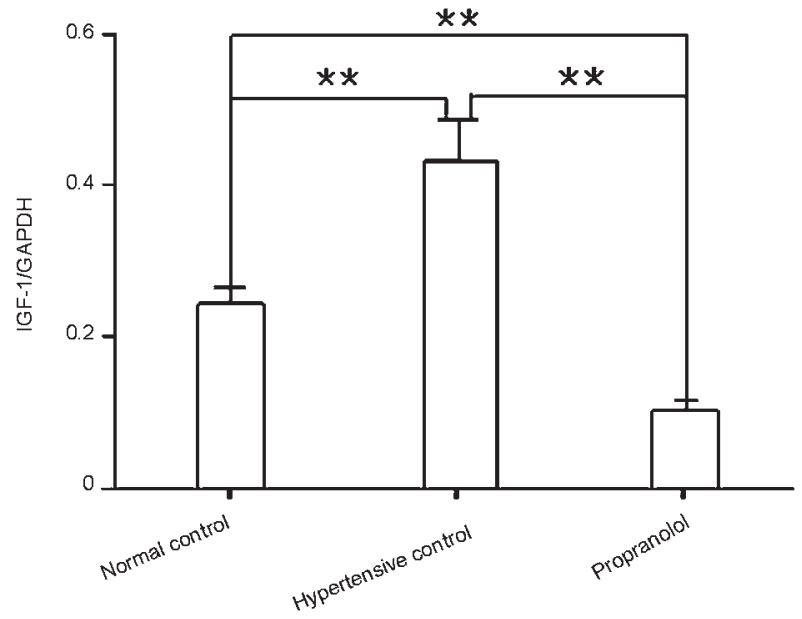

Figure 4 Propranolol decreases IGF-1 mRNA expression in rat cavernous tissue. Quantitative real time-PCR analysis of IGF-1 mRNA levels in cavernous tissue was done 4 weeks after propranolol treatment. The data were collected from nine rats in each group. $* * P<0.01$. GAPDH, glyceraldehyde 3-phosphate dehydrogenase; IGF, insulin-like growth factor.
Figure 5 Propranolol decreases IGF-1 protein expression in rat cavernous tissue. Western blot analysis of IGF protein expression in cavernous tissue from normal control (lane 1), hypertensive control (lane 2) and propranolol-treated rats (lane 3) was done 4 weeks after propranolol treatment. The data were collected from nine rats in each group. $* * P<0.01$. GAPDH, glyceraldehyde 3-phosphate dehydrogenase; IGF, insulin-like growth factor.

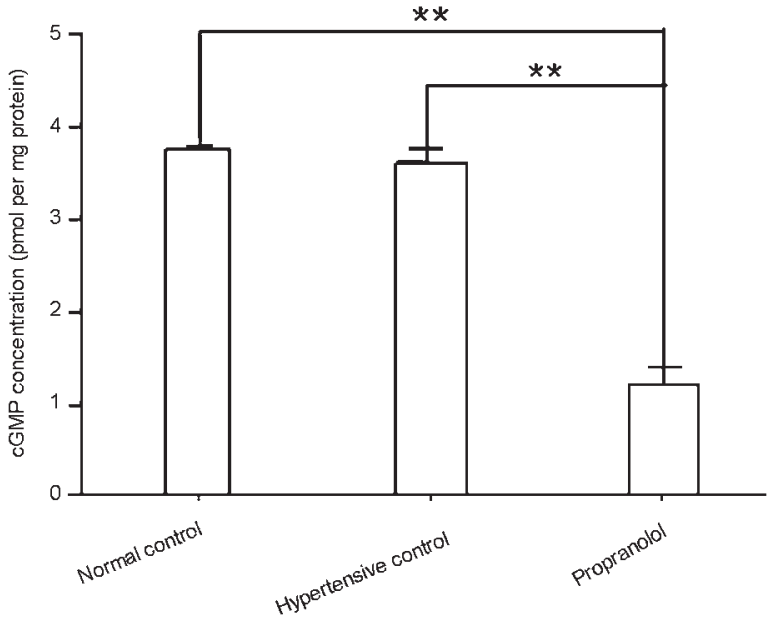

Figure 6 Propranolol treatment decreases the cGMP concentration in rat cavernous tissue. CGMP concentration in rat penile tissue 4 weeks after propranolol treatment, determined by ELISA. The data were collected from nine rats in each group. $* * P<0.01$. cGMP, cyclic guanosine monophosphate; ELISA, enzymelinked immunosorbent assay. 
to IGFBP-3. This results in decreased availability of IGF-1 for the receptor. ${ }^{8}$ IGF-1 has been shown to enhance the regeneration of nitric oxide synthase-containing penile nerves after cavernous neurotomy and to enhance the recovery of erectile function after bilateral cavernous nerve cryoablation. ${ }^{13}$ It has also been shown that $\beta$-blockers, including propranolol, can induce IGFBP-3 production. ${ }^{14}$ Thus, we speculate that propranolol could enhance the production of IGFBP-3, which then contributes to $\mathrm{ED}$ in $2 \mathrm{~K}-1 \mathrm{C}$ hypertensive rats treated by propranolol, by decreasing the availability of IGF-1. However, additional experiments will be required to demonstrate that IGF-1 availability is reduced in the hypertensive rat penis; we will address this in future studies.

The relaxation of corporal smooth muscle (CSM) is essential for normal erectile function, and substantial evidence implicates the NOcGMP signalling pathway as the principal mediator of CSM relaxation and penile erection. ${ }^{15} \mathrm{NO}$ released in response to sexual stimulation relaxes penile vascular smooth muscle by increasing the intracellular cGMP concentration. ${ }^{16}$ The vasodilatation of erectile tissue allows the sinusoidal spaces to fill with blood, resulting in the attainment and maintenance of an erection. ${ }^{17}$ One previous study has shown that enhanced levels of contracting factors in hypertension impaired the NO-cGMP signalling pathway and promoted ED. ${ }^{18}$ Hypertension might protect against ED in the initial stages of chronic arterial insufficiency. ${ }^{19}$ However, later on, hypertension has contradictory effects on ED. On the one hand, it worsens ED as a result of chronic arterial insufficiency due to the progression of atherosclerosis; on the other hand, it overcomes the obstruction of penile artery stenosis in the initial stages of the disease by directly interfering with pulse pressure. ${ }^{19}$ Our findings suggest that decreased cGMP concentration may contribute to $\mathrm{ED}$ in the penile tissue of the $2 \mathrm{~K}-1 \mathrm{C}$ hypertensive rats after propranolol treatment.

Here, we provide several lines of evidence that the increased expression of IGFBP-3 might play a role in the development of $\mathrm{ED}$ in $2 \mathrm{~K}-1 \mathrm{C}$ hypertensive rats 4 weeks after propranolol treatment. We further show that this is associated with decreased IGF-1 availability and downregulation of NO-cGMP signalling in the penis. Further studies are necessary to explore the mechanistic link between IGFBP-3 upregulation and the downregulation of $\mathrm{NO}-$ cGMP signalling.

\section{AUTHOR CONTRIBUTIONS}

$\mathrm{ZYZ}$ and XHW conducted the molecular genetic studies, participated in the sequence alignment and drafted the manuscript. DC and YZW carried out the immunoassays. ZHY participated in the sequence alignment. HHZ, MP and QLL participated in the design of the study and performed the statistical analyses. HC and SPW conceived the study, participated in its design and coordination and helped to draft the manuscript. All authors read and approved the final manuscript.

\section{COMPETING FINANCIAL INTERESTS}

The authors declare no competing financial interests.

\section{ACKNOWLEDGMENTS}

We thank Prof. B. W. Peng and Dr S. R. Guo for their excellent technical support. This study was supported by the National Natural Science Foundation of China (no. 30872572).

1 Lue TF. Erectile dysfunction. N Engl J Med 2000; 342: 1802-13.

2 Fogari R, Zoppi A, Corradi L, Mugellini A, Poletti L et al. Sexual function in hypertensive males treated with lisinopril or atenolol: a cross-over study. Am J Hypertens 1998; 11: 1244-7.

3 Shabsigh R, Perelman MA, Lockhart DC, Lue TF, Broderick GA. Health issues of men: prevalence and correlates of erectile dysfunction. J Urol 2005; 174: 662-7.

4 Cervenka L, Horacek V, Vaneckova I, Hubacek JA, Oliverio MI et al. Essential role of AT1A receptor in the development of $2 \mathrm{~K} 1 \mathrm{C}$ hypertension. Hypertension 2002; 40 : 735-41.

5 Lazartigues E, Lawrence AJ, Lamb FS, Davisson RL. Renovascular hypertension in mice with brain-selective overexpression of AT1a receptors is buffered by increased nitric oxide production in the periphery. Circ Res 2004; 95: 523-31.

6 Hiyoshi H, Yayama K, Takano M, Okamoto H. Angiotensin type 2 receptor-mediated phosphorylation of eNOS in the aortas of mice with 2-kidney, 1-clip hypertension. Hypertension 2005; 45: 967-73.

7 Linder AE, Bendhack LM. Endothelin-1-induced contraction is impaired in the tail artery of renal hypertensive rats. Vascul Pharmacol 2002; 39: 77-82.

8 Soh J, Katsuyama M, Ushijima S, Mizutani Y, Kawauchi A et al. Localization of increased insulin-like growth factor binding protein-3 in diabetic rat penis: implications for erectile dysfunction. Urology 2007; 70: 1019-23.

9 Pacca SR, de Azevedo AP, de Oliveira CF, de Luca IM, de Nucci G et al. Attenuation of hypertension, cardiomyocyte hypertrophy, and myocardial fibrosis by betaadrenoceptor blockers in rats under long-term blockade of nitric oxide synthesis. J Cardiovasc Pharmacol 2002; 39: 201-7.

$10 \mathrm{Pu} X Y$, Hu LQ, Wang HP, Luo YX, Wang XH. Improvement in erectile dysfunction after insulin-like growth factor-1 gene therapy in diabetic rats. Asian J Androl 2007; 9: 83-91.

11 Vorwerk P, Oh Y, Lee PD, Khare A, Rosenfeld RG. Synthesis of IGFBP-3 fragments in a baculovirus system and characterization of monoclonal anti-IGFBP-3 antibodies. J Clin Endocrinol Metab 1997; 82: 2368-70.

12 Kasper JS, Liu Y, Pollak MN, Rifai N, Giovannucci E. Hormonal profile of diabetic men and the potential link to prostate cancer. Cancer Causes Control 2008. 19: 703-10.

13 Bochinski D, Hsieh PS, Nunes L, Lin GT, Lin CS et al. Effect of insulin-like growth factor- 1 and insulin-like growth factor binding protein-3 complex in cavernous nerve cryoablation. Int J Impot Res 2004; 16: 418-23.

14 Jeschke MG, Finnerty CC, Kulp GA, Przkora R, Mlcak RP et al. Combination of recombinant human growth hormone and propranolol decreases hypermetabolism and inflammation in severely burned children. Pediatr Crit Care Med 2008; 9: 209-16.

15 Bivalacqua TJ, Usta MF, Kendirci M, Pradhan L, Alvarez X et al. Superoxide anion production in the rat penis impairs erectile function in diabetes: influence of in vivo extracellular superoxide dismutase gene therapy. J Sex Med 2005; 2: 187-97.

16 Lamina S, Okoye CG, Dagogo TT. Managing erectile dysfunction in hypertension: the effects of a continuous training programme on biomarker of inflammation. BJU Int 2009; 103: 1218-21.

17 Watts GF, Chew KK, Stuckey BG. The erectile-endothelial dysfunction nexus: new opportunities for cardiovascular risk prevention. Nat Clin Pract Cardiovasc Med 2007; 4: 263-73.

18 Gur S, Kadowitz PJ, Gurkan L, Chandra S, Dewitt SY et al. Chronic inhibition of nitricoxide synthase induces hypertension and erectile dysfunction in the rat that is not reversed by sildenafil. BJU Int 2010; 106: 78-83.

19 Spessoto LC, Cordeiro JA, de Godoy JM. Effect of systemic arterial pressure on erectile dysfunction in the initial stages of chronic arterial insufficiency. BJU Int 2010; 106: 1723-5. 